\title{
Design of Collagen-Based Sponge Device for Use in Oral Surgery
}

\author{
Yoshimitsu Kuroyanagi ${ }^{1,2 *}$, Ryusuke Suzuki ${ }^{3}$, Misato Kuroyanagi ${ }^{4}$ \\ ${ }^{1} \mathrm{R} \& D$ Center for Skin Regenerative Medicine, Technosurg Ltd., Sagamihara, Japan \\ ${ }^{2}$ School of Allied Health Sciences, Kitasato University, Sagamihara, Japan \\ ${ }^{3}$ SHEM, Inc., Tokyo, Japan \\ ${ }^{4}$ Department of Emergency Medicine and Department of Plastic and Reconstructive Surgery, Graduate School of Medicine, \\ Yokohama City University, Yokohama, Japan \\ Email: *drs.yoshi@technosurg.co.jp
}

How to cite this paper: Kuroyanagi, Y., Suzuki, R. and Kuroyanagi, M. (2021) Design of Collagen-Based Sponge Device for Use in Oral Surgery. Open Journal of Regenerative Medicine, 10, 31-49.

https://doi.org/10.4236/ojrm.2021.103003

Received: August 1, 2021

Accepted: September 27, 2021

Published: September 30, 2021

Copyright $\odot 2021$ by author(s) and Scientific Research Publishing Inc. This work is licensed under the Creative Commons Attribution International License (CC BY 4.0).

http://creativecommons.org/licenses/by/4.0/

\begin{abstract}
This study aims to develop various types of collagen devices for use in oral surgery. The targets are the sheet-shaped sponges to cover mucosal defect wounds (product-1) and gingival defect wounds (product-2) and the cylindrical sponge to fill tooth extraction sockets (product-3). The sheet-shaped sponges were manufactured by freeze-vacuum drying the aqueous solution of collagen (Col) and heat-denatured collagen (Col') at a composition ratio of $2 / 1,1 / 1$ or $1 / 2$. Both surfaces of the sheet-shaped sponge were treated by ultraviolet (UV) irradiation for 5, 10 or 15 minutes to introduce intermolecular crosslinks between collagen molecules. The elution behavior of each collagen sponge was investigated by immersing the sponge in water for a predetermined time and then by measuring the weight change. The collagen sponge composed of Col and Col' with a composition ratio of $2 / 1$ that was treated by UV irradiation for 15 minutes showed very slow elution properties. This sheet-shaped sponge is the top candidate for product-1. The collagen sponge composed of Col and Col' with a composition ratio of $2 / 1$ that was treated by UV irradiation for 5 minutes showed slightly fast elution properties. This sheet-shaped sponge is the top candidate for product-2. Next, the cylindrical sponge was manufactured by freeze-vacuum drying the aqueous solution of $\mathrm{Col}$ and Col' at a composition ratio of $2 / 1$. Both sides of the cylindrical sponge were treated by UV irradiation for 15 minutes. This sponge showed initially fast elution properties and subsequent very slow elution properties. This cylindrical sponge is a good candidate for product-3. As a basic design, the sheet-shaped sponge and the cylindrical sponge should be biodegraded and absorbed by the time new tissue formation is completed.
\end{abstract}




\section{Keywords}

Collagen Sponge Device, Mucosal Wound Dressing, Gingival Wound

Dressing, Tooth Extraction Socket Filler

\section{Introduction}

Several types of wound dressings composed of collagen (Col) have been developed and used in the field of plastic and reconstructive surgery [1] [2] [3]. One of the excellent wound dressings is the sheet-shaped collagen sponge with a silicone film attached to the top surface [4]. This wound dressing is manufactured by freeze-vacuum drying a mixed aqueous solution of fibrous Col and heat-denatured collagen ( $\mathrm{Col}$ '), followed by thermal dehydration to introduce intermolecular crosslinks between collagen molecules. Fibrous $\mathrm{Col}$ is prepared by neutralizing an acidic $\mathrm{Col}$ aqueous solution with phosphate buffer. The fibrous $\mathrm{Col}$ in the sponge serves as a scaffold for new tissue formation. The Col' in the sponge acts as a wound healing promoter. In the treatment of severe burns, this wound dressing is applied to deep tissue defect wounds after excision of necrotic tissue. Under good conditions, blood vessels and fibroblasts invade the inside of the sponge from the surrounding dermis to form granulation tissue. The treatment with this wound dressing can form excellent transplant beds for autologous split-thickness skin grafts. The function of fibrous Col as a scaffold is considered to be important for extensive and deep tissue defect wounds.

Wound dressings are also needed in the field of oral surgery. The sheet-shaped collagen sponge with the same composition as the wound dressing described above was developed. The treatments in oral surgery using this collagen sponge were reported [5] [6] [7] [8]. Some properties to be improved have been pointed out for this collagen sponge. In the treatment of root surface exposure due to gingival degeneracy and loss of attached gingiva, mucosal fragments are collected from palatal mucosa and then transplanted to gingival defect wounds. The sheet-shaped collagen sponge can be applied to mucosal defect wounds caused by collecting mucosal fragments. Under good conditions, blood vessels and mucosal fibroblasts invade the inside of the sponge from the surrounding mucosa to form granulation tissue. At the same time as this process, the surrounding mucosal epithelium divides, proliferates, and moves on this granulation tissue to form epidermis. Since the area of mucosal defect wounds is small, it is possible to form epidermis from the surroundings. Alternatively, the sheet-shaped collagen sponge can be applied to gingival defect wounds. Under good conditions, blood vessels and gingival fibroblasts invade the inside of the sponge from the surrounding gingiva to form granulation tissue. At the same time as this process, the surrounding gingival epithelium divides, proliferates, and moves on this granulation tissue to form epidermis. Since the area of gingival defect wounds is small, it is possible to form epidermis from the surroundings. Basically, the 
treatment of small and shallow tissue defect wounds in the oral cavity is different from the treatment of extensive and deep tissue defect wounds in burn injury. Therefore, a new design suitable for collagen sponge used in oral surgery is needed.

In addition, the collagen sponge devise is also needed for the treatment of tooth extraction sockets. The cylindrical collagen sponge with the same composition as the wound dressing described above was developed. The treatments of tooth extraction socket using this cylindrical sponge were reported [9] [10]. In this case as well, some properties to be improved have been pointed out for this collagen sponge. In the normal wound healing process, blood clots form in the tooth extraction socket. Blood vessels and gingival fibroblasts invade the inside of the blood clot to form granulation tissue. At the same time as this process, the surrounding gingival epithelium divides, proliferates, and moves on this granulation tissue to form epidermis. Under good conditions, osteoblasts emerge inside the granulation tissue and alveolar bone regenerates. Since the space of the tooth extraction socket is small, it is important to maintain the space for cell migration in the sponge combined with blood clot. As a basic design, the sheetshaped sponge and the cylindrical sponge should be biodegraded and absorbed by the time new tissue formation is completed.

In our previous study, the wound dressing composed of hyaluronic acid and Col was developed [11] [12] [13] [14]. Hyaluronic acid has an excellent wound healing promoting function, but has a poor hemostatic function [15] [16]. The sponge devices used in the field of oral surgery need to have a hemostatic effect, because it is not easy to stop bleeding in the oral cavity. Therefore, this study focused on the design of sponge devices composed of Col. It is well known that $\mathrm{Col}$ is an important biomaterial for wound healing as it has various biological activities [17]. In addition, Col has an excellent hemostatic function [18]. Col molecules are considered to have a better wound healing function in the nonfibrous state than in the fibrous state [19]. From this point of view, we designed the sponge devices composed of non-fibrous Col and Col'. The purpose of this study is to develop various types of collagen sponge devices used in the field of oral surgery. The targets are the sheet-shaped sponges to cover mucosal defect wounds (product-1) and gingival defect wounds (product-2) and the cylindrical sponge to fill tooth extraction sockets (product-3).

\section{Materials and Methods}

\subsection{Manufacture of Sheet-Shaped Sponge to Cover Mucosal and Gingival Defect Wounds}

Purified granular collagen (Col) derived from porcine skin was purchased from Nippon Meat Packers (Osaka, Japan). Terror peptides of collagen molecule that are antigenic determinants have been removed by enzyme degradation. Col (21.6 g) was dissolved in distilled water $(1800 \mathrm{~mL})$ under stirring with a screw propeller below $25^{\circ} \mathrm{C}$ overnight to obtain $\mathrm{Col}$ aqueous solution (1.2 w/v\%, $\mathrm{pH} 2.8$ ). 
Aside from this, Col $(21.6 \mathrm{~g})$ was dissolved in distilled water $(1800 \mathrm{~mL})$ under stirring with a screw propeller below $25^{\circ} \mathrm{C}$ overnight and then heated at $60^{\circ} \mathrm{C}$ for 1 hour to obtain heat-denatured collagen aqueous solution ( $1.2 \mathrm{w} / \mathrm{v} \%, \mathrm{pH} 2.8)$. This heat-denatured collagen is referred to as Col'. The Col aqueous solution was mixed with the Col' aqueous solution by stirring and then adjusted to the conditions of $\mathrm{pH} 4.5$ by dropping sodium hydroxide aqueous solution to obtain a clear mixture.

First, the Col aqueous solution $(800 \mathrm{ml})$ was mixed with the Col' aqueous solution $(400 \mathrm{~mL})$. This mixture $(2 \mathrm{~mL})$ was poured into each of the 10 partitions $(18 \mathrm{~mm} \times 18 \mathrm{~mm})$ of a plastic tray and refrigerated at $4^{\circ} \mathrm{C}$ for 6 hours or more, and then was frozen at $-85^{\circ} \mathrm{C}$ overnight and followed by freeze-vacuum drying to obtain the sheet-shaped sponge. This sheet-shaped sponge was named as Sponge-CC'21. The composition ratio of $\mathrm{Col}$ and Col' is $2 / 1$. Second, the $\mathrm{Col}$ aqueous solution $(600 \mathrm{~mL})$ was mixed with the Col' aqueous solution $(600 \mathrm{~mL})$. The sheet-shaped sponge was manufactured in a similar way. This sponge was named as Sponge-CC'11. The composition ratio of $\mathrm{Col}$ and Col' is $1 / 1$. Finally, the $\mathrm{Col}$ aqueous solution $(400 \mathrm{ml})$ was mixed with the Col' aqueous solution $(800 \mathrm{~mL})$. The sheet-shaped sponge was manufactured in a similar way. This sponge was named as Sponge-CC'12. The composition ratio of Col and Col' is $1 / 2$.

Both surfaces of the sheet-shaped sponge were irradiated using a $15 \mathrm{~W}$ ultraviolet lamp with a wavelength of $253.7 \mathrm{~nm}$ at a distance of $20 \mathrm{~cm}$ to introduce intermolecular crosslinks between collagen molecules. Both surfaces of SpongeCC' 21 were treated by UV irradiation for 5, 10, 15 minutes. They were named as Sponge-CC'21-5x2, Sponge-CC'21-10x2 and Sponge-CC'21-15x2, respectively. Both surfaces of Sponge-CC'11 were treated by UV irradiation for 5, 10, 15 minutes. They were named as Sponge-CC'11-5x2, Sponge-CC'11-10x2 and SpongeCC'11-15x2, respectively. Both surfaces of Sponge-CC'12 were treated by UV irradiation for 5, 10, 15 minutes. They were named as Sponge-CC'12-5x2, SpongeCC'12-10x2 and Sponge-CC'12-15x2, respectively.

\subsection{Manufacture of Cylindrical Sponge to Fill Tooth Extraction Sockets}

The cylindrical sponge was manufactured in the same way as the sheet-shaped sponge. Col $(8.0 \mathrm{~g})$ was dissolved in distilled water $(400 \mathrm{~mL})$ to obtain Col aqueous solution $(2.0 \mathrm{w} / \mathrm{v} \%, \mathrm{pH} 2.8)$. Aside from this, $\mathrm{Col}(4.0 \mathrm{~g})$ was dissolved in distilled water $(200 \mathrm{~mL})$ and then heated at $60^{\circ} \mathrm{C}$ for 1 hour to obtain Col' aqueous solution $(2.0 \mathrm{w} / \mathrm{v} \%, \mathrm{pH} 2.8)$. The $\mathrm{Col}$ aqueous solution was mixed with the Col' aqueous solution by stirring and then adjusted to the conditions of $\mathrm{pH} 4.5$ by dropping sodium hydroxide aqueous solution to obtain a clear mixture. This mixture $(2 \mathrm{~mL})$ was poured into each of the cylindrical shaped plastic bottle with a diameter of $10 \mathrm{~mm}$ and a depth of $30 \mathrm{~mm}$ and refrigerated at $4^{\circ} \mathrm{C}$ for 6 hours or more, and then was frozen at $-85^{\circ} \mathrm{C}$ overnight and followed by freeze-vacuum 
drying to obtain the cylindrical sponge. This sponge was named as cy-SpongeCC'21. The composition ratio of $\mathrm{Col}$ and Col' is $2 / 1$. Both sides of the cylindrical sponge were treated by UV irradiation for 15 minutes. This sponge was named as cy-Sponge-CC'21-15x2.

\subsection{Water Absorption of Sponge when Immersed in Water}

The water absorption of each sheet-shaped sponge was measured by immersing 10 sheet-shaped sponges in a plastic container filled with distilled water (200 $\mathrm{mL}$ ) at $37^{\circ} \mathrm{C}$. After 10 minutes, the hydrated sponges were collected using a 10-mesh size stainless steel mesh and then placed on this mesh for 30 minutes to remove excess water and then weighed. The weight of 10 hydrated sponges was measured and compared with the original weight before immersion in water. Each experiment was performed using 8 containers. The average value was calculated by measuring the weight of the hydrated 10 sponges collected from each container. In a similar way, the water absorption of the cylindrical sponge was measured by immersing 5 cylindrical sponges in a plastic container filled with distilled water $(200 \mathrm{~mL})$ at $37^{\circ} \mathrm{C}$. After 10 minutes, the hydrated sponges were collected using a 10-mesh size stainless steel mesh and then placed on this mesh for 30 minutes to remove excess water and then weighed. The weight of 5 hydrated sponges was measured and compared with the original weight before immersion in water. This experiment was performed using 8 containers. The average value was calculated by measuring the weight of the hydrated 5 sponges collected from each container.

\subsection{Weight Change of Sheet-Shaped Sponge when Immersed in Water}

The weight change of sheet-shaped sponge was measured by immersing 10 sheet-shaped sponges in a plastic container filled with distilled water $(200 \mathrm{~mL})$ and placing it in an incubator at $37^{\circ} \mathrm{C}$ for a predetermined time. The hydrated 10 sponges were collected using a 10-mesh size stainless steel mesh after immersion in water for 3 hour, 6 hour, 12 hour, 1 day, 2 days, 3 days, 5 days and 7 days and then returned to a plastic tray $(80 \mathrm{~mm} \times 50 \mathrm{~mm})$. These hydrated sponges were frozen at $-85^{\circ} \mathrm{C}$ overnight and followed by freeze-vacuum drying to obtain dry sponges. The weight of 10 dry sponges was measured and compared with the original weight before immersion in water. Each experiment was performed using 8 containers. The average value was calculated by measuring the weight of 10 dry sponges collected from each container.

\subsection{Weight Change of Cylindrical Sponge when Immersed in Water}

The weight change of cylindrical sponge was measured by immersing 5 cylindrical sponges in a plastic container filled with distilled water $(200 \mathrm{~mL})$ and placing it in an incubator at $37^{\circ} \mathrm{C}$ for a predetermined time. The hydrated 5 
sponges were collected using a 10-mesh size stainless steel mesh after immersion in water for 3 hour, 6 hour, 12 hour, 1 day, 3 days and 7 days and then returned to a plastic tray $(80 \mathrm{~mm} \times 50 \mathrm{~mm})$. These hydrated sponges were frozen at $-85^{\circ} \mathrm{C}$ overnight, and followed by freeze-vacuum drying to obtain dry sponges. The weight of 5 dry sponges was measured and compared with the original weight before immersion in water. This experiment was performed using 4 containers. The average value was calculated by measuring the weight of 5 dry sponges collected from each container.

\subsection{Enzymatic Degradation of Sponge by Collagenase}

Collagenase powder was purchased from FUJIFILM Wako Pure Chemical Corporation (Osaka, Japan). The enzymatic degradation of sheet-shaped sponge by collagenase was investigated using Sponge-CC'21-15x2, Sponge-CC'11-15x2 and Sponge-CC'12-15x2 as typical samples. Collagenase (32 mg) was dissolved in distilled water $(1600 \mathrm{~mL})$. The enzyme concentration was adjusted to 0.002 $\mathrm{w} / \mathrm{v} \%$. The weight change of each sheet-shaped sponge was measured by immersing 10 sheet-shaped sponges in each plastic container filled with distilled water containing collagenase $(200 \mathrm{~mL})$ and placing it in an incubator at $37^{\circ} \mathrm{C}$. The hydrated sponges were collected using a 10-mesh size stainless steel mesh after immersion for 1 day and then returned to a plastic tray $(80 \mathrm{~mm} \times 50 \mathrm{~mm})$. These hydrated sponges were frozen at $-85^{\circ} \mathrm{C}$ overnight and followed by freezevacuum drying to obtain dry sponges. The weight of 10 dry sponges was measured and compared with the original weight. Each experiment was performed using 8 containers. The average value was calculated by measuring the weight of 10 dry sponges collected from each container.

In addition, the enzymatic degradation of cylindrical sponge by collagenase was measured using cy-Sponge-CC'21-15x2 in a similar way. The weight change of this cylindrical sponge was measured by immersing 5 cylindrical sponges in each container filled with distilled water containing collagenase $(200 \mathrm{~mL})$ and placing it in an incubator at $37^{\circ} \mathrm{C}$. The hydrated sponges were collected using a 10 -mesh size stainless steel mesh after immersion for 1 day and then returned to a plastic tray $(80 \mathrm{~mm} \times 50 \mathrm{~mm})$. These hydrated sponges were frozen at $-85^{\circ} \mathrm{C}$ overnight and followed by freeze-vacuum drying to obtain dry sponges. The weight of 5 dry sponges was measured and compared with the original weight. This experiment was performed using 8 containers. The average value was calculated by measuring the weight of 5 dry sponges collected from each container.

\subsection{Statistical Evaluation}

Data were expressed as means \pm standard error. Statistical analysis was performed using Student's t-test for comparison between two groups. Each experiment was performed 8 times to examine statistically significant differences in the measured values $(n=8)$. 


\section{Result}

\subsection{Manufacture of Sheet-Shaped Sponge to Cover Mucosal and Gingival Defect Wounds}

As a preliminary experiment, the change in viscosity of Col aqueous solution due to temperature change was measured. Col dissolves in water under acidic conditions of $\mathrm{pH} 3$ or less. However, precipitation occurs when $\mathrm{pH}$ value reaches 4 or higher under $25^{\circ} \mathrm{C}$ conditions. Such a phenomenon can be considered as follows. The positively charged state of the side chains of Col molecules weakens at $\mathrm{pH} 4$ or higher. As a result, it is thought that the binding force between the side chains of the Col molecules and the surrounding water molecules is weakened. Such conditions promote lateral alignment between rod-shaped Col molecules having a triple helical structure, thereby causing aggregation and precipitation. Once the acidic Col aqueous solution is heated to $40^{\circ} \mathrm{C}$ or higher, Col molecules undergo a conformational change from triple helical structure to random structure. This phenomenon reflects a decrease in viscosity. The viscosity of $\mathrm{Col}$ aqueous solution was measured at the range from $20^{\circ} \mathrm{C}$ to $60^{\circ} \mathrm{C}$ using a standard viscometer. This viscometer rotates the cylindrical part in the solution and measures the rotating top surface resistance. The results of preliminary experiments showed that the viscosity of $\mathrm{Col}$ aqueous solution $(1.2 \mathrm{w} / \mathrm{v} \%)$ was $10.0,9.2,0.1$ and $0.1 \mathrm{dPa} \cdot \mathrm{s}$ at $20^{\circ} \mathrm{C}, 30^{\circ} \mathrm{C}, 40^{\circ} \mathrm{C}$ and $60^{\circ} \mathrm{C}$, respectively. Once the $\mathrm{Col}$ aqueous solution was heated to $60^{\circ} \mathrm{C}$ and then cooled to $20^{\circ} \mathrm{C}$, the viscosity was $0.4 \mathrm{dPa} \cdot \mathrm{s}$ (Figure 1). This phenomenon indicates that the random structure does not return to the original helical structure. Such conditions inhibit lateral alignment between random-shaped molecules due to mutual steric hindrance. As a result, this heat-denatured Col' aqueous solution does not precipitate even

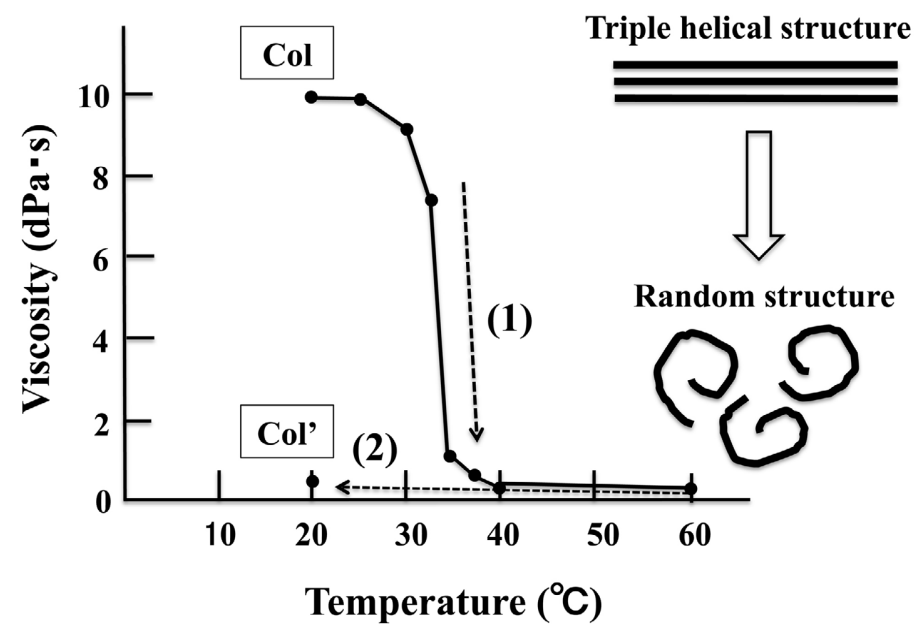

Figure 1. Change in viscosity of Col aqueous solution due to temperature change: 1) Significant decrease in viscosity due to temperature change from $30^{\circ} \mathrm{C}$ to $40^{\circ} \mathrm{C}$, indicating conformational change from triple helical structure to random structure. 2) No increase in viscosity due to temperature change from $60^{\circ} \mathrm{C}$ to $20^{\circ} \mathrm{C}$, indicating no conformational change from random structure to triple helical structure. 
in the neutral $\mathrm{pH}$ range. The mixed aqueous solution of Col and Col' with a composition ratio of $1 / 2$ causes partial precipitation at $\mathrm{pH} 5.5$ or higher. The mixed aqueous solution of Col and Col' with a composition ratio of $1 / 1$ or $2 / 1$ causes partial precipitation at $\mathrm{pH} 5.0$ or higher. For this reason, the $\mathrm{pH}$ of these mixed aqueous solutions was set to 4.5 to obtain a clear mixture.

The sheet-shaped sponges were manufactured by freeze-vacuum drying this clear mixed aqueous solution of $\mathrm{Col}$ and Col' under the condition of $1.2 \mathrm{w} / \mathrm{v} \%$ collagen concentration at $\mathrm{pH}$ 4.5. The both surfaces of sheet-shaped sponge were irradiated by UV lamp to introduce crosslinks between collagen molecules. Sponge-CC'21 and Sponge-CC'11 are very soft and are suitable for application to soft mucosal and gingival defect wounds. On the other hand, Sponge-CC'12 is harder than other two sponges and is not suitable for application to soft mucosal and gingival defect wounds. The sheet-shaped sponge has a size of $17 \mathrm{~mm} \times 17$ $\mathrm{mm}$ and a thickness of $3 \mathrm{~mm}$ (Figure 2(a)).

\subsection{Manufacture of Cylindrical Sponge to Fill Tooth Extraction Sockets}

The cylindrical sponge was manufactured by freeze-vacuum drying the mixed aqueous solution of Col and Col' under the condition of $2.0 \mathrm{w} / \mathrm{v} \%$ collagen concentration at $\mathrm{pH}$ 4.5. The cylindrical sponge shrank slightly after freeze-vacuum drying. The sponge could be easily removed from the cylindrical shaped plastic bottle container. This cy-Sponge-CC'21 showed sufficient physical properties to be used as filler for tooth extraction sockets. A small mark was placed on one part of the cylindrical sponge and then UV irradiation was performed on both sides to introduce crosslinks between collagen molecules. The cylindrical sponge has a diameter of $8 \mathrm{~mm}$ and a length of $25 \mathrm{~mm}$ (Figure 2(b)). The diameter of

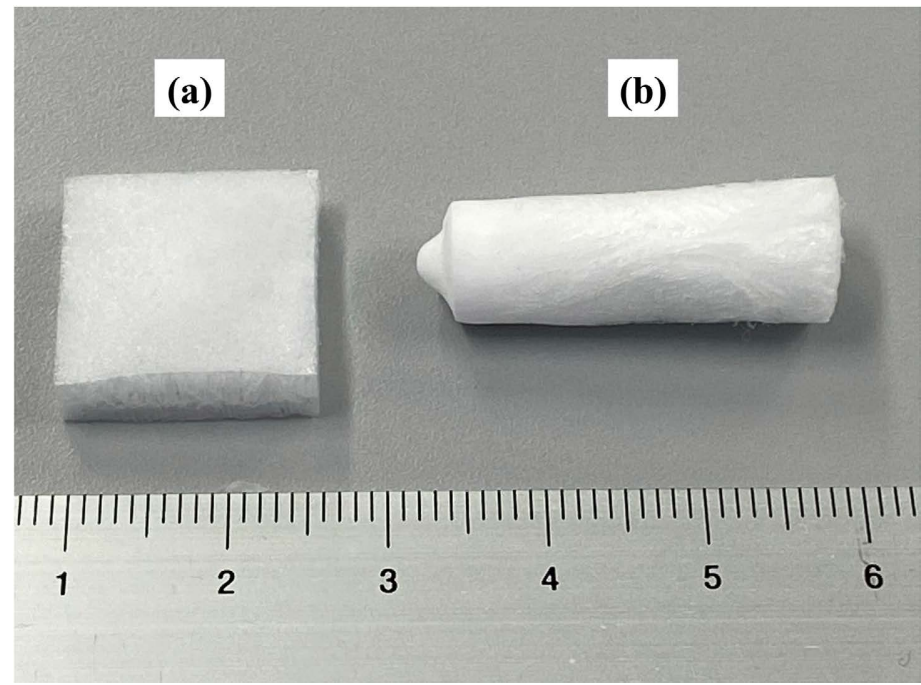

Figure 2. Appearances of sheet-shaped sponge and cylindrical sponge: (a) Sponge-CC’21-15x2 has a size of $17 \mathrm{~mm} \times 17 \mathrm{~mm}$ and a thickness of $3 \mathrm{~mm}$. (b) cy-Sponge-CC'21-15x2 has a diameter of $8 \mathrm{~mm}$ and a length of $25 \mathrm{~mm}$. 
the cylindrical sponge is matched to the diameter of tooth extraction socket. This sponge is inserted into the tooth extraction socket and compressed to match both size.

\subsection{Water Absorption of Sponge when Immersed in Water}

It is important that the sponge has high water absorption properties. It is a great merit to be able to absorb a large amount of exudates containing various cell growth factors. For this reason, the water absorption properties of collagen sponges were examined. Sheet-shaped sponges showed high water absorption when immersed in water at $37^{\circ} \mathrm{C}$ for 10 minutes (Figure 3). Sponge-CC'12-15x2 absorbed 45 times more water than its own weight. Sponge-CC'11-15x2 absorbed 48 times more water than its own weight. Sponge-CC'21-15x2 absorbed 46 times more water than its own weight. On the other hand, cy-Sponge-CC'21$15 \times 2$ absorbed 30 times more water than its own weight. The sheet-shaped sponge was manufactured by freeze-vacuum drying the collagen aqueous solution at the concentration of $1.2 \mathrm{w} / \mathrm{v} \%$. On the contrary, the cylindrical sponge was manufactured by freeze-vacuum drying the collagen aqueous solution at the concentration of $2.0 \mathrm{w} / \mathrm{v} \%$. The cylindrical sponge has a denser structure than the sheet-shaped sponge. As a result, cy-Sponge-CC’21-15x2 showed a lower water absorption value than Sponge-CC'21-15x2.

\subsection{Weight Change of Sheet-Shaped Sponge when Immersed in Water}

It is important that collagen sponge is biodegraded and absorbed by the time

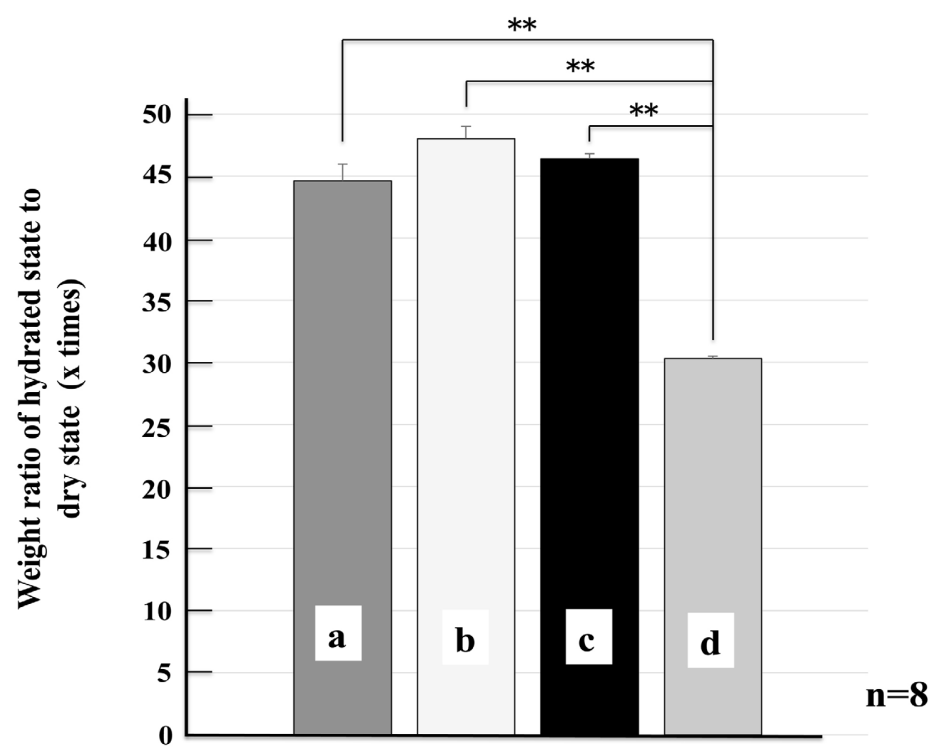

Figure 3. Water absorption properties of sheet-shaped sponge and cylindrical sponge when immersed in water: Weight ratio of hydrated state to dry state (x times). (a) Sponge-CC'12-15x2; (b) Sponge-CC'11-15x2; (c) Sponge-CC’21-15x2; (d) cy-Sponge-CC’21-15x2. ${ }^{* *} \mathrm{P}<0.01$. [Student's t-test]. 
new tissue formation is completed. The weight change of the sponge when immersed in water is related to the degree of crosslinks between collagen molecules. The degree of crosslinks is related to UV irradiation time. For this reason, the weight changes of collagen sponges treated by different UV irradiation times were examined. The weight change of the sheet-shaped sponge was measured by immersing 10 sheet-shaped sponges in water at $37^{\circ} \mathrm{C}$ (Figure $4(\mathrm{a})$ ). The weight change rate of Sponge-CC'12 when immersed in water was measured. The weight change rate of Sponge-CC'12-15x2 was $70 \%$ of the original weight when immersed in water for 7 days. On the contrary, the weight change rate of SpongeCC' $12-5 \times 2$ was $32 \%$ of the original weight when immersed in water for 3 days (Figure 5). Next, the weight change of Sponge-CC'11 when immersed in water was measured. The weight change rate of Sponge-CC'11-15x2 was $71 \%$ of the original weight when immersed in water for 7 days. On the contrary, the weigh change rate of Sponge-CC'11-5x2 was $25 \%$ of the original weight when immersed in water for 3 days (Figure 6). Next, the weight change of Sponge-CC'21 when immersed in water was measured. The weight change rate of SpongeCC'21-15x2 was $76 \%$ of the original weight when immersed in water for 7 days. On the contrary, the weight change rate of Sponge-CC'21-5x2 was $51 \%$ of the original weight when immersed in water for 3 days (Figure 7). These results indicate that the degree of crosslinks between collagen molecules depends on the UV irradiation time.

The degree of crosslinks between collagen molecules also depended on the composition ratio of Col and Col'. The intermolecular crosslinks was inadequate under the condition of UV irradiation time of 5 minutes, and increased in the

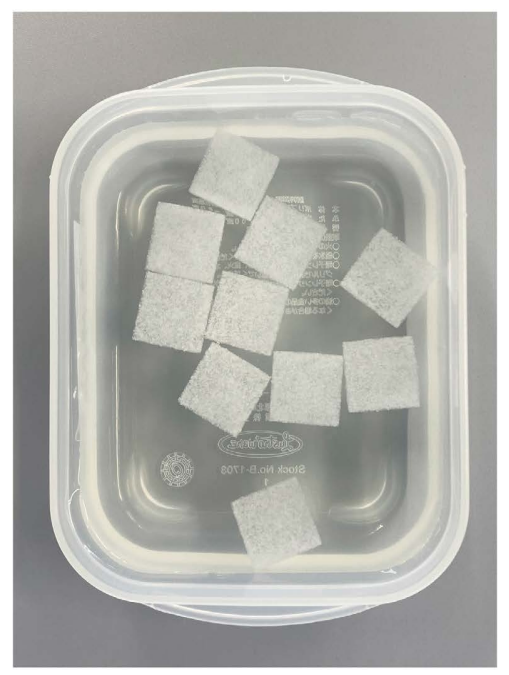

(a) Sponge-CC'21-15x2

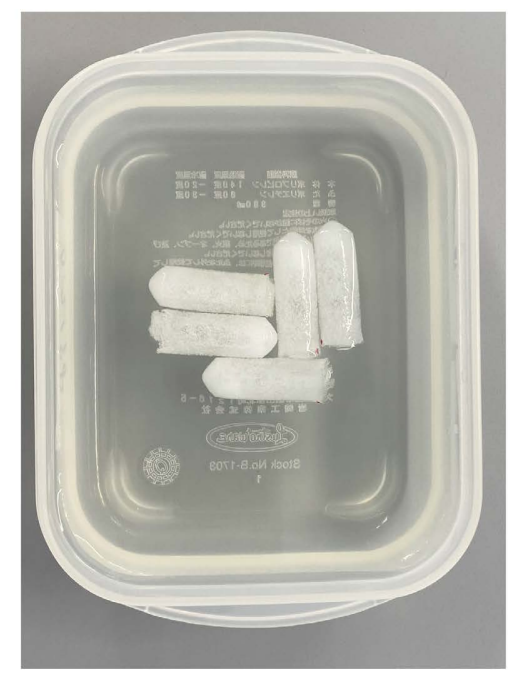

(b) cy-Sponge-CC'21-15x2

Figure 4. Experimental system for measuring weight changes of sheet-shaped sponge and cylindrical sponge: (a) 10 pieces of Sponge-CC'21-15x2 were immersed in distilled water $(200 \mathrm{~mL})$. This experiment was performed using 8 containers. (b) 5 pieces of cy-Sponge-CC’21-15x2 were immersed in distilled water $(200 \mathrm{~mL})$. This experiment was performed using 4 containers. 


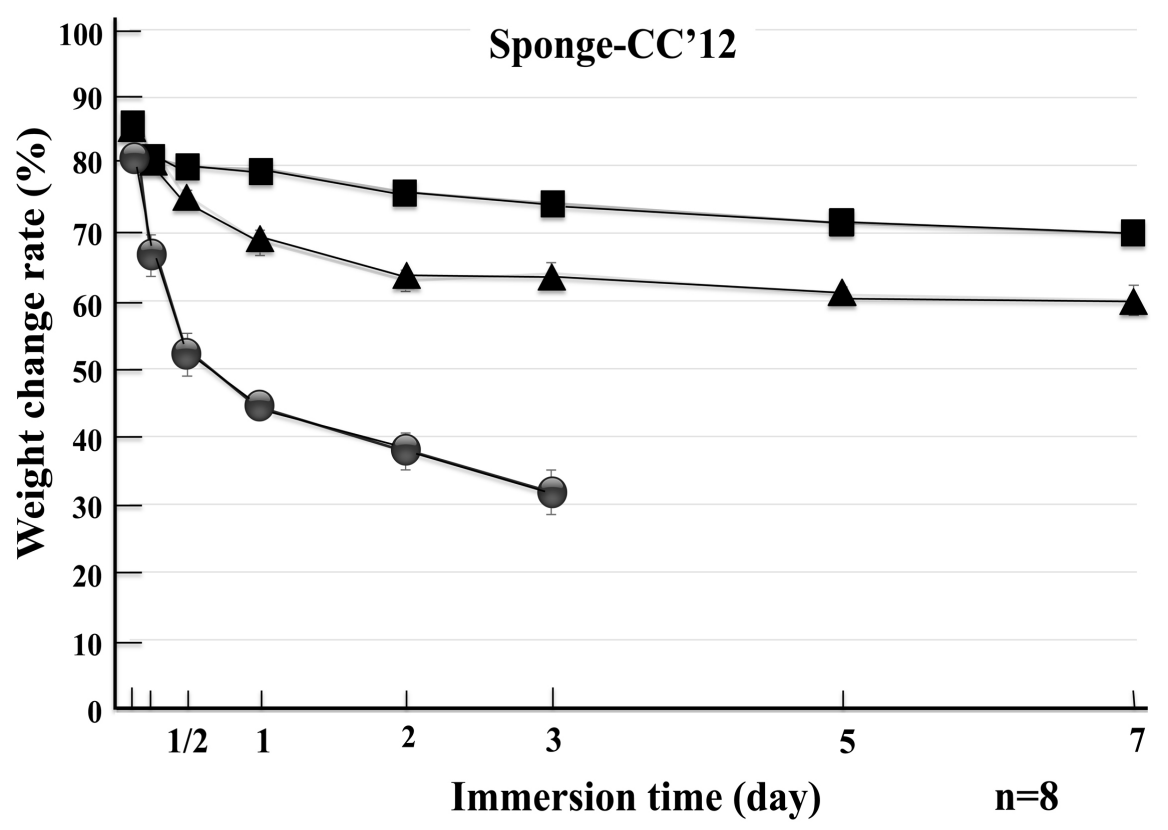

Figure 5. Weight change rate (\%) of Sponge-CC'12 when immersed in water: $(\bullet)$ Sponge-CC'12-5x2; ( $\Delta$ ) Sponge-CC'12-10x2; (घ) Sponge-CC'12-15x2.

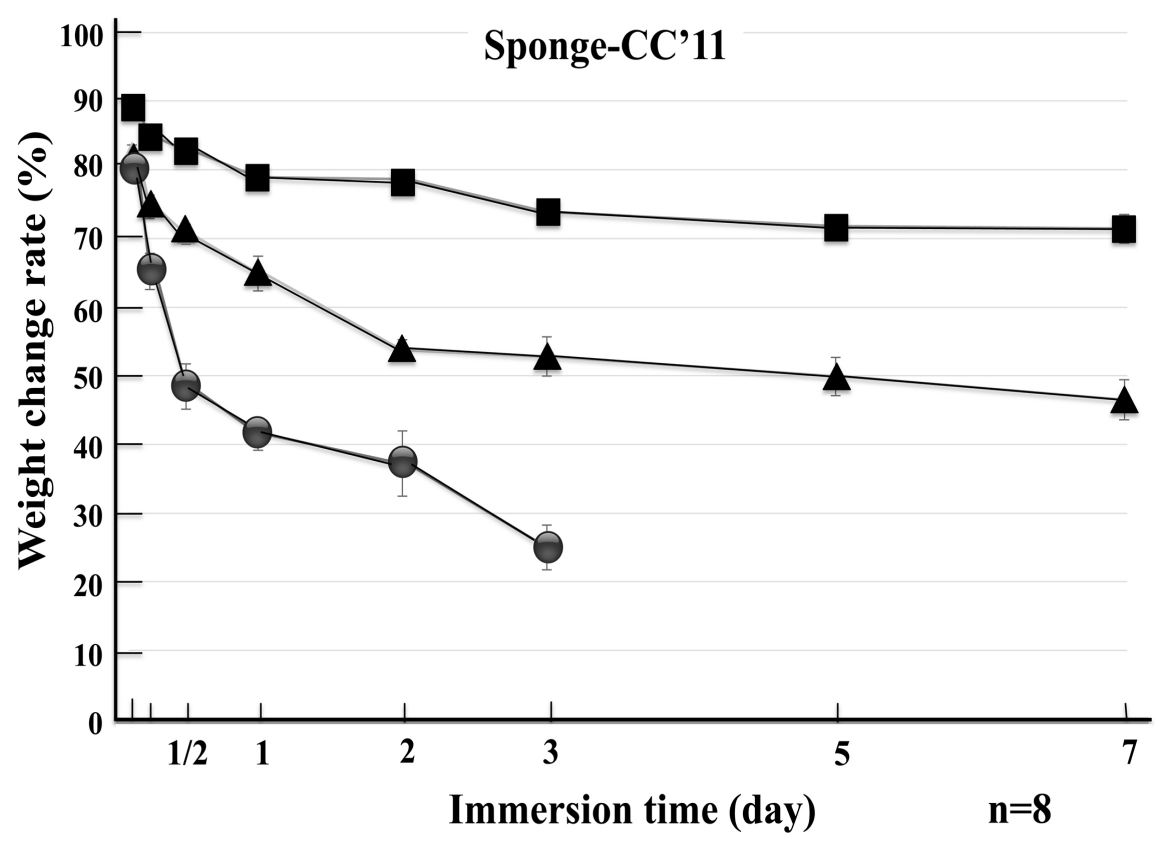

Figure 6. Weight change rate $(\%)$ of Sponge-CC'11 when immersed in water: $(\bullet)$ Sponge-CC'11-5x2; ( $\Delta$ ) Sponge-CC'11-10x2; (घ) Sponge-CC'11-15x2.

order of Sponge-CC'11-5x2, Sponge-CC'12-5x2, and Sponge-CC'21-5x2 (Figure 8). The intermolecular crosslinks was successfully introduced under the condition of UV irradiation time of 10 minutes, and increased in the order of SpongeCC'11-10x2, Sponge-CC'12-10x2, and Sponge-CC'21-10x2 (Figure 9). There was a slight difference in weight change among 3 groups due to sufficient intermolecular crosslinks when UV irradiation time was 15 minutes (Figure 10). 


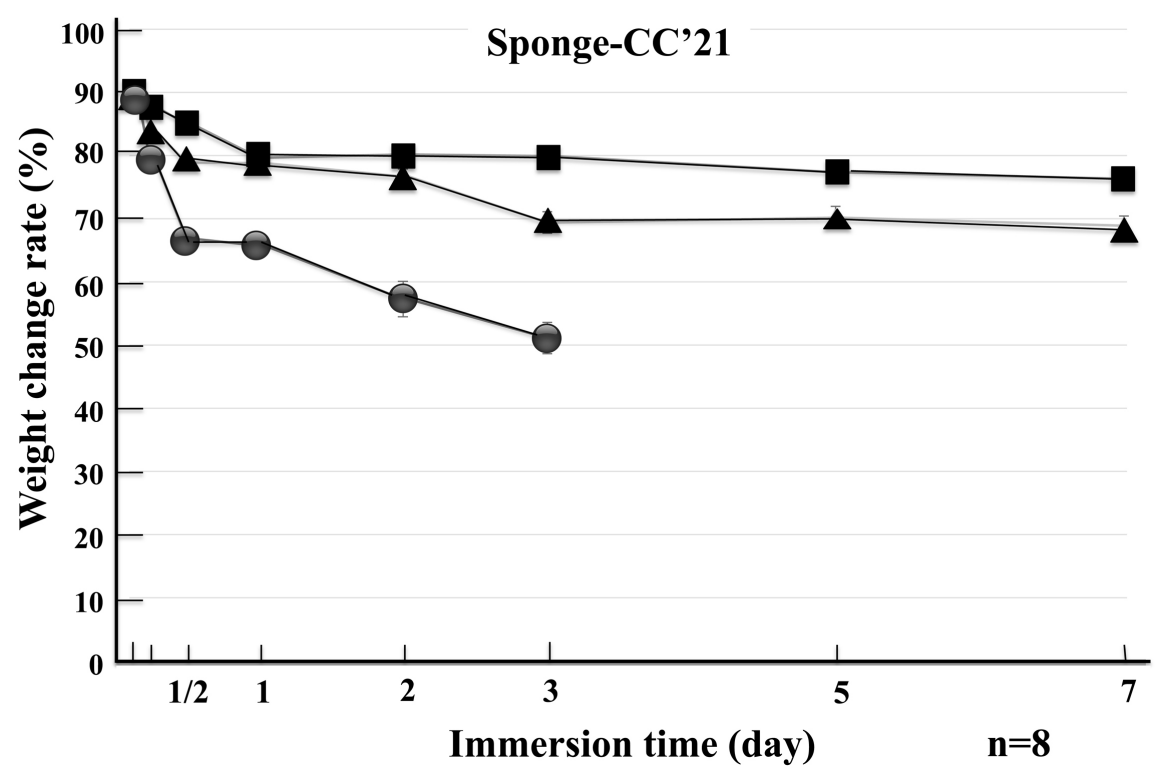

Figure 7. Weight change rate (\%) of Sponge-CC'21 when immersed in water: (•) Sponge-CC'21-5x2; ( $\mathbf{\Delta}$ ) Sponge-CC’21-10x2; (घ) Sponge-CC’21-15x2.

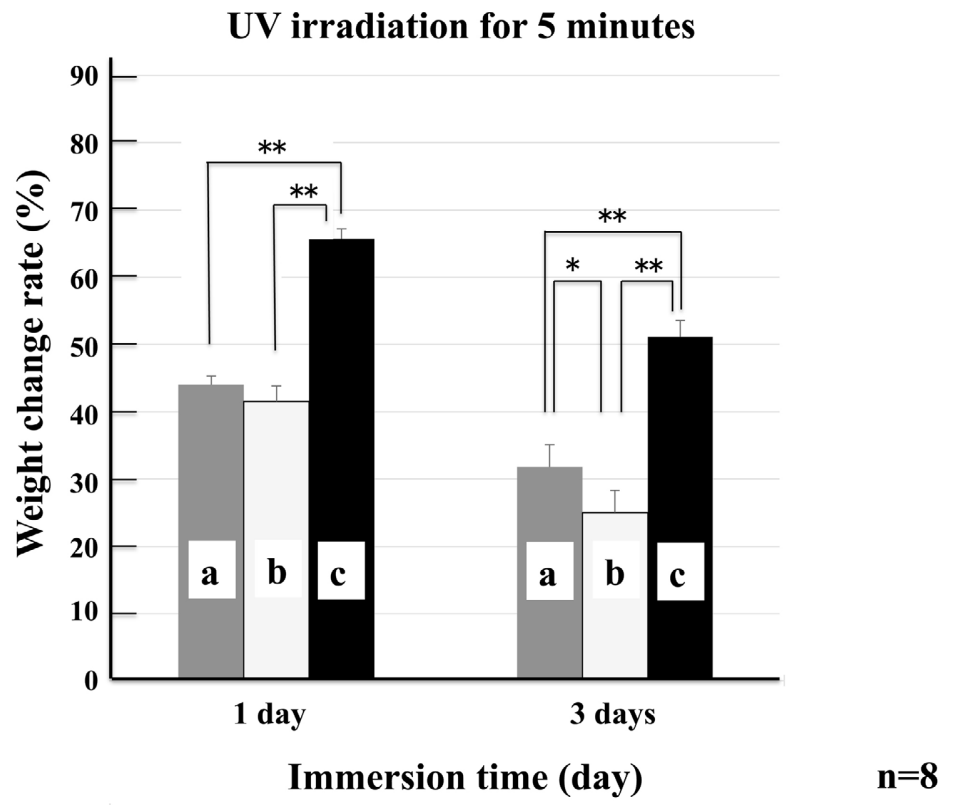

Figure 8. Comparison of weight change rate (\%) of sheet-shaped sponges treated by UV irradiation for 5 minutes: (a) Sponge-CC'12-5x2; (b) Sponge-CC'11-5x2; (c) Sponge-CC’21-5x2; ${ }^{\star} \mathrm{P}<0.1 ;{ }^{*} \mathrm{P}<0.01$. [Student's t-test].

\subsection{Weight Change of Cylindrical Sponge when Immersed in Water}

The weight change of cylindrical sponge was measured by immersing 5 cylindrical sponges in water at $37^{\circ} \mathrm{C}$ (Figure $4(\mathrm{~b})$ ). The weight change rate of cySponge-CC'-21-15x2 measured 3 hours, 6 hours, 12 hours, 1 day, 3 days and 7 days after immersion was $88 \%, 72 \%, 49 \%, 44 \%, 36 \%$ and $34 \%$ of the original 


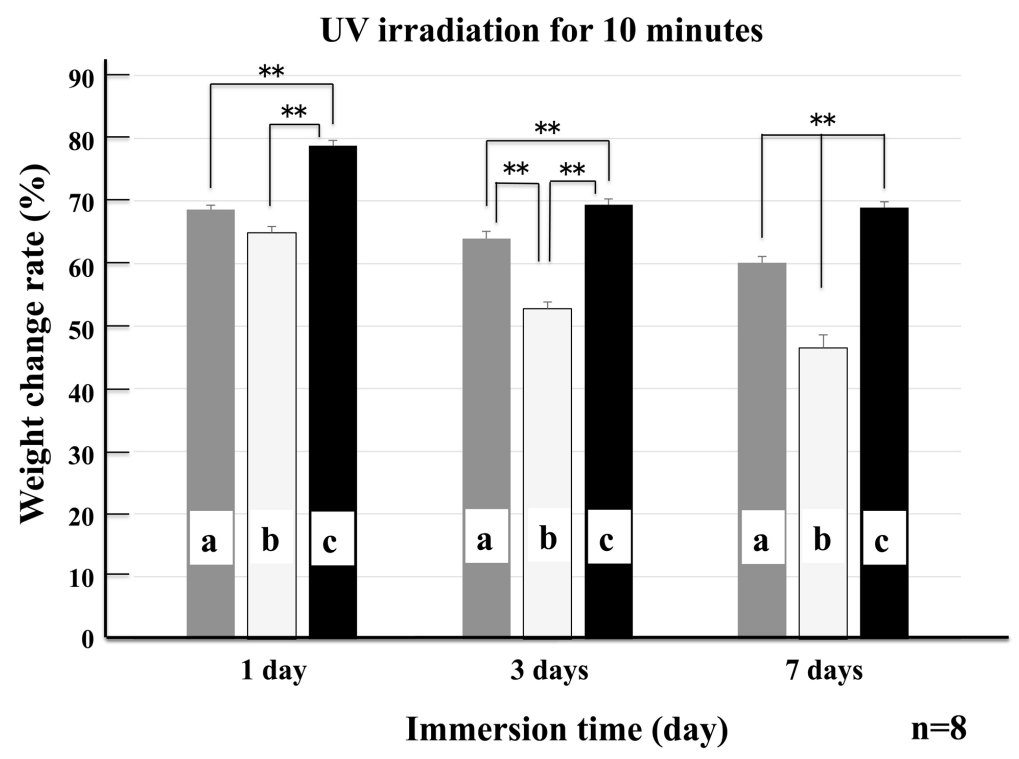

Figure 9. Comparison of weight change rate (\%) of sheet-shaped sponges treated by UV irradiation for 10 minutes: (a) Sponge-CC'12-10x2; (b) SpongeCC’11-10x2; (c) Sponge-CC’21-10x2. ${ }^{*} \mathrm{P}<0.01$. [Student's t-test].

UV irradiation for 15 minutes

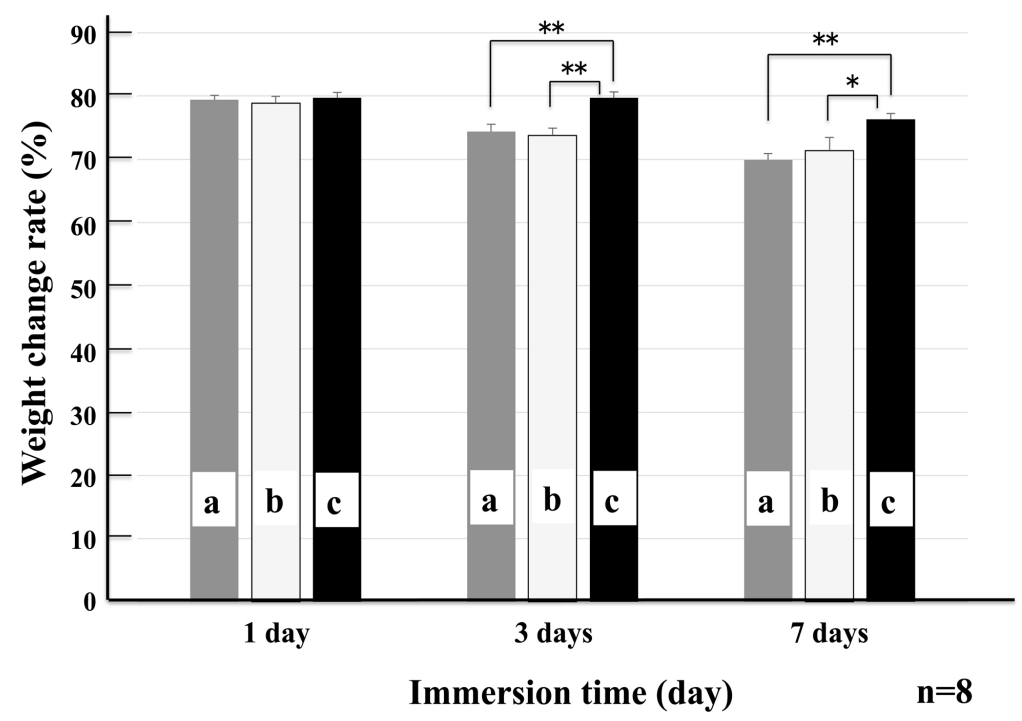

Figure 10. Comparison of weight change rate (\%) of sheet-shaped sponges treated by UV irradiation for 15 minutes: (a) Sponge-CC'12-15x2; (b) SpongeCC'11-15x2; (c) Sponge-CC'21-15x2. ${ }^{\star} \mathrm{P}<0.1,{ }^{\star *} \mathrm{P}<0.01$. [Student's t-test].

weight, respectively (Figure 11). The cylindrical sponge showed a marked weight loss 12 hours after immersion in water, thereafter a slight weight lost.

\subsection{Enzymatic Degradation of Sponge by Collagenase}

It is important that collagen sponge is biodegraded and absorbed by the time new tissue formation is completed. As an in vitro experiment, the enzymatic degradation properties of sponge by collagenase were investigated. The enzymatic 
degradation of sheet-shaped sponge and cylindrical sponge was determined (Figure 12). As a result of comparative experiment, the weight change rate of Sponge-CC'12-15x2, Sponge-CC'11-15x2 and Sponge-CC’21-15x2 when immersed in water for 1 day was $79 \%, 79 \%$ and $80 \%$ of the original weight, respectively. The weight change rate of Sponge-CC'12-15x2, Sponge-CC'11-15x2 and Sponge-CC'21-15x2 when immersed in water containing collagenase $(0.002$

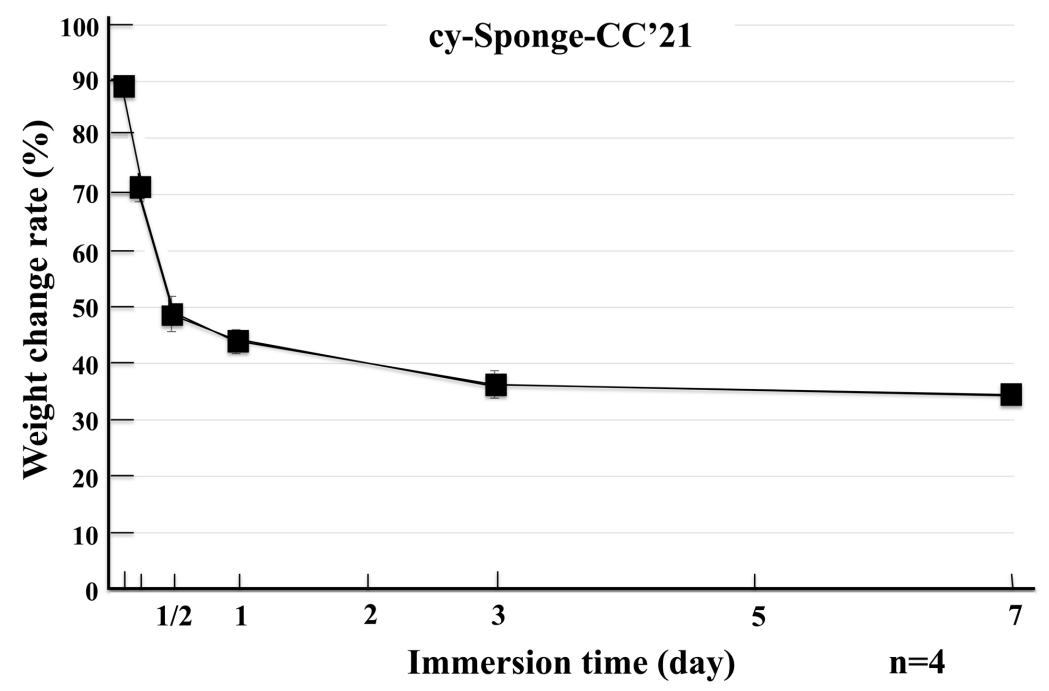

Figure 11. Weight change rate (\%) of cy-Sponge-CC'21 when immersed in water: (匹) cy-Sponge-CC'21-15x2.

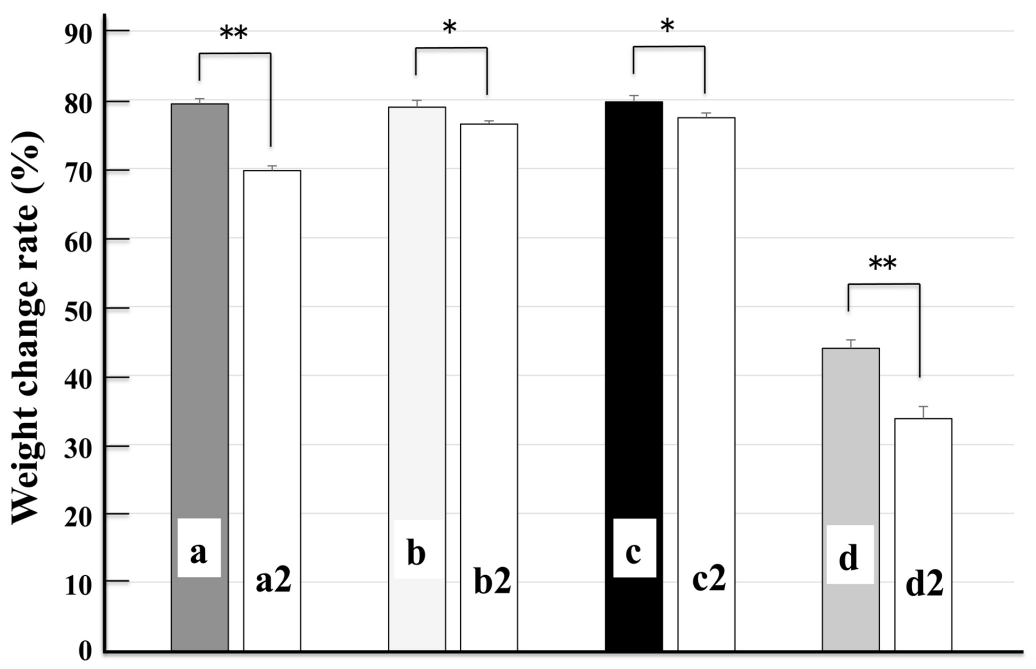

Enzymatic degradation by collagenase $\quad n=8$

Figure 12. Enzymatic degradation behavior of sheet-shaped sponge and cylindrical sponge by collagenase: Weight change rate (\%) of sponge when immersed in water for 1 day: (a) Sponge-CC'12-15x2; (b) Sponge-CC'11-15x2; (c) SpongeCC'21-15x2; (d) cy-Sponge-CC’21-15x2. Weigh change rate (\%) of sponge when immersed in water contacting collagenase $(0.002 \mathrm{w} / \mathrm{v} \%)$ for 1 day: (a2) SpongeCC'12-15x2; (b2) Sponge-CC'11-15x2; (c2) Sponge-CC'21-15x2; (d2) cy-SpongeCC'21-15x2. ${ }^{\star} \mathrm{P}<0.1,{ }^{* *} \mathrm{P}<0.01$, [Student's t-test]. 
w/v\%) for 1 day was $70 \%, 77 \%$ and $77 \%$ of the original weight, respectively. This result suggests that the crosslinks between collagen molecules in these sheetshaped sponges can effectively delay enzymatic degradation by collagenase. In addition, the weight change rate of cy-Sponge-CC'21-15x2 when immersed in water for 1 day was $44 \%$ of the original weight. On the other hand, the weight change rate of cy-Sponge-CC'21-15x2 when immersed in water containing collagenase $(0.002 \mathrm{w} / \mathrm{v} \%)$ was $34 \%$ of the original weight. This result suggests that the crosslinks between collagen molecules in this cylindrical sponge also can effectively delay enzymatic degradation by collagenase. It is expected that collagen sponge is biodegraded and absorbed by the time new tissue formation is completed.

\section{Discussion}

What should be emphasized is the following. Basically, the treatment of small and shallow tissue defect wounds in the oral cavity is different from the treatment of extensive and deep tissue defect wounds in burn injury. In the former healing process, the function of non-fibrous $\mathrm{Col}$ as providing space for cell $\mathrm{mi}$ gration is considered to be important. In the later healing process, the function of fibrous Col as a scaffold is considered to be important. Therefore, a new design suitable for collagen sponge used in oral surgery is needed.

This study focused on the following two ideas. The first important idea in the manufacturing process is how to prepare a clear mixed aqueous solution of Col and Col'. The second important idea in the manufacturing process is how to control the crosslinks between collagen molecules. The general method for introducing crosslinks between collagen molecules is to use chemical cross-linking agents. This method requires the removal of unreacted cross-linking agents. This process is disadvantageous in manufacturing products. One practical method is the introducing crosslinks by thermal dehydration in a vacuum at $110^{\circ} \mathrm{C}$ for several hours. Another practical method is the introducing crosslinks by UV irradiation. We adopted the method of introducing crosslinks by UV irradiation. This method has the advantage that the degree of crosslinks can be controlled arbitrarily.

In this study, we investigated the optimum composition ratio of Col and Col' and the optimum conditions for introducing crosslinks between collagen molecules. We examined the weight change of sheet-shaped sponges when immersed in water. As typical comparison data, the weight change rate of Sponge-CC'$21-15 \times 2$ when immersed in water for 3 days and 7 days were $80 \%$ and $76 \%$ of the original weight before immersion in water. On the other hand, the weight change rate of Sponge-CC'21-5x2 when immersed in water for 3 days was $51 \%$ of the original weight before immersion in water. It is considered that SpongeCC'21-15x2 was cross-linked sufficiently in the outer layer but insufficiently in the intermediate layer of the sponge, whereas Sponge-CC'21-5x2 was crosslinked insufficiently in both outer layer and intermediate layer of the sponge. 
The sheet-shaped sponge for covering mucosal defect wounds (product-1) and the sheet-shaped sponge for covering gingival defect wounds (product-2) require different properties. The product- 1 needs to protect mucosal defect wounds for at least two weeks. Sponge-CC'21-15x2 is the top candidate for product-1, since it has very slow elution properties. The product- 2 is inserted inside the incised gingiva. It is desirable to be mostly biodegraded and absorbed within a week. Sponge-CC'21-5x2 is the top candidate for product-2, since it has slightly fast elution properties.

The tooth extraction sockets have less exudate than mucosal and gingival defect wounds. The filler for extraction socket is desirable to be mostly biodegraded and absorbed within two weeks. It is considered that cy-Sponge-CC'21-15x2 has a unique structure. The collagen molecules in the outer layer were sufficiently cross-linked, whereas the collagen molecules in the central part were insufficiently cross-linked. When immersed in water, some molecules in the central part quickly dissolve into water and then gradually dissolve into water. It is expected that a sustained outflow of collagen molecules from the sponge can create an environment for cell migration. The cy-Sponge-CC’21-15x2 is a good candidate for product-3, since it has initially fast elution properties and subsequent very slow elution properties.

When the sheet-shaped sponge (product-1 and product-2) or cylindrical sponge (product-3) is applied to wounds, it is considered that the internal insufficiently cross-linked collagen molecules elute first, and then the external sufficiently cross-linked collagen molecules elute with the collapse of the reticulated structure due to enzymatic degradation. Such a sustained outflow of collagen molecules from the sponge creates an environment that promotes cell migration (Figure 13). One of the factors that create such an environment is a physical effect of voids created by elution of collagen molecules from the sponge. Another is a biological effect that eluted collagen molecules act as chemotactic factors to attract surrounding fibroblasts to the wound site. As a basic design, the sheet-shaped sponge and the cylindrical sponge should be biodegraded and absorbed by the time new tissue formation is completed.

In addition to the above two manufacturing ideas, there is a practical idea for clinical use. As mentioned above, the excellent wound dressing is a collagen sponge with a silicone film attached to the top surface [4]. In the treatment of severe burns, this wound dressing is attached to necrotic tissue excision wounds, and fixed easily with a normal bandage. In the treatment of oral mucosal and gingival defect wounds, such a wound dressing must be secured by suturing the perimeter. Actually, it is difficult to prevent infection from the sutured part. To solve this shortcoming, we propose a new fixing method using a tissue adhesive. In our previous study, it was demonstrated that PMMA-based tissue adhesive has moderate viscosity and instant adhesiveness, and is effective in closing skin incisions [20]. This tissue adhesive is a product for oral surgery. An effective method is possible by using collagen sponge and PMMA-based tissue adhesive 


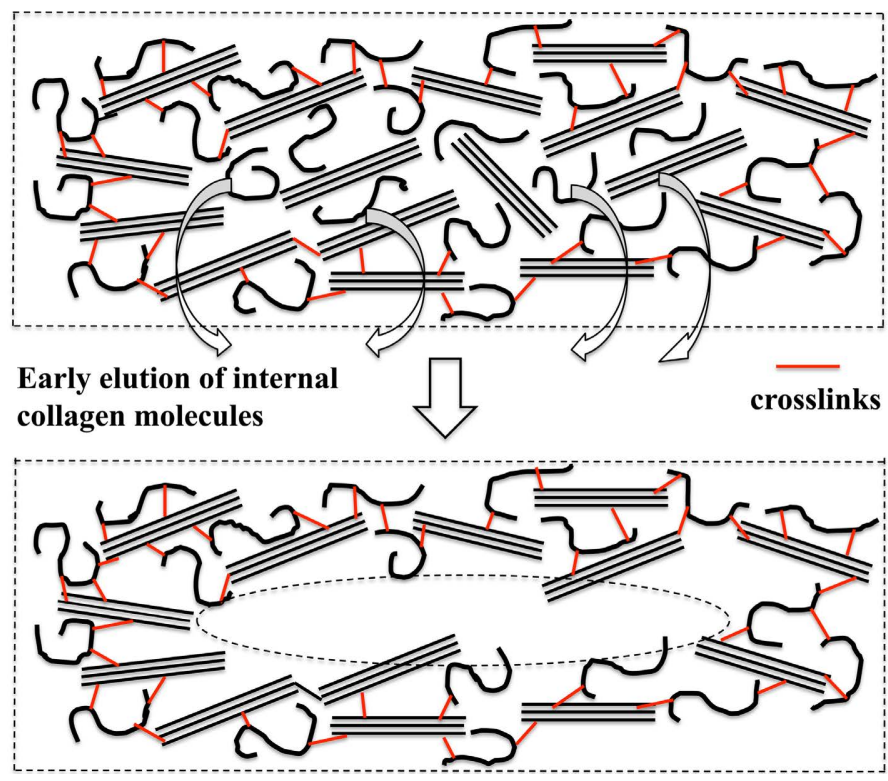

Figure 13. Schematic diagram of both sided UV irradiated sheet-shaped sponge: Internal insufficiently cross-linked collagen molecules elute first, and then the external sufficiently cross-linked collagen molecules elute with the collapse of the reticulated structure due to enzymatic degradation.

together. A sheet-shaped sponge is attached to mucosal and gingival defect wounds, and then PMMA-based tissue adhesive is coated on it to fix it. Covering the outer surface and surroundings of the sheet-shaped sponge with this tissue adhesive has the advantage of preventing infection. Similarly, a cylindrical sponge is filled into tooth extraction socket, and then PMMA-based tissue adhesive is coated on it to fix it. Covering the top of the cylindrical sponge with this tissue adhesive has the advantage of preventing infection.

Comprehensively, it is important to consider technological progress and practicality. Tissue-engineering products composed of cells and biomaterials have been developed for root coverage [21] [22]. In our previous study, autologous cultured gingival dermis substitute composed of gingival fibroblasts and matrix has been developed [23]. This autologous cultured product was demonstrated to be a beneficial grafting material for root coverage in the treatment of gingival recession. This tissue-engineered product has excellent performance but is not practical due to high manufacturing cost and long manufacturing time. On the contrary, the sheet-shaped collagen sponge developed in this study is more practical due to its high performance and low manufacturing cost. This sheet-shaped collagen sponge is practical because it can be used immediately after being taken out of the sterilization bag.

\section{Conclusion}

The sheet-shaped sponge and the cylindrical sponge were manufactured by freeze-vacuum drying the aqueous solution of $\mathrm{Col}$ and $\mathrm{Col}^{\prime}$ at a composition ra- 
tio of 2/1. The both sides of sponge were treated with UV irradiation for 5, 10, 15 minutes to introduce crosslinks between collagen molecules. As a basic design, the sheet-shaped sponge and the cylindrical sponge should be biodegraded and absorbed by the time new tissue formation is completed. Sponge-CC'21-10x2 is the top candidate for the mucosal wound dressing. Sponge-CC'21-5x2 is the top candidate for the gingival wound dressing. In addition, cy-Sponge-CC'21-15x2 is a good candidate for the tooth extraction socket filler.

\section{Conflicts of Interest}

The authors declare no conflicts of interest regarding the publication of this paper.

\section{References}

[1] Burke, J.F., Yannas, I.V., Quinby, W.C., Bondoc, C.C. and Jung, W.K. (1981) Successful Use of a Physiologically Acceptable Artificial Skin in the Treatment of Extensive Burn Injury. Annals of Surgery, 194, 413-428. https://doi.org/10.1097/00000658-198110000-00005

[2] Yannas, I.V., Burke, J.F., Orgill, D.P. and Skrabut, E.M. (1982) Wound Tissue Can Utilize a Polymeric Template to Synthesize a Functional Extension of Skin. Science, 215, 174-176. https://doi.org/10.1126/science.7031899

[3] Suzuki, S., Matsuda, K., Isshiki, N., Tamada, Y., Yoshioka, K. and Ikada, Y. (1990) Clinical Evaluation of a New Bilayer Artificial Skin Composed of Collagen Sponge and Silicone Layer. British Journal of Plastic Surgery, 43, 47-54. https://doi.org/10.1016/0007-1226(90)90044-Z

[4] Koide, M., Osaki, K., Konishi, J., Oyamada, K., Katakura, T., Takahashi, A. and Yoshizato, K. (1993) A New Type of Biomaterial for Artificial Skin: Dehydrothermally Cross-Linked Composites of Fibrillar and Denatured Collagen. Journal of Biomedical Material Research, 27, 79-87. https://doi.org/10.1002/jbm.820270111

[5] Mizuki, N., Omura, S., Aoki, S., Umino, S., Kawabe, R., Ishikawa, Y., Ono, S. and Fujita, K. (1994) Clinical Evaluation of a New Collagen Sponge and Silicone Bilayer "Artificial Mucous Membrane” for the Repair of Oral Mucosal Defect. Japanese Journal of Oral and Maxillofacial Surgery, 40, 776-784. https://doi.org/10.5794/jioms.40.776

[6] Shirai, Y., Bessho, K., Nisida, M., Murakami, K. and Iizuka, T. (1995) The Usefulness of a Bilayer Artificial Dermis (TERUDERMIS®) for Vestibular Extension in the Mandible. Japanese Journal of Oral and Maxillofacial Surgery, 41, 896-898. https://doi.org/10.5794/jijoms.41.896

[7] Fushimi, H., Kodama, T., Tsutsumi, K., Kitsugi, D., Sugiyama, T., Ikai, H. and Hori, T. (1997) The Effect of Artificial Dermis (Terudermis () Applied to Muco-Gingival Surgery -Clinical Trial-. Japanese Society of Oral Implantology, 10, 45-54.

[8] Omura, S., Mizuki, N., Horimoto, S., Kawabe, R. and Fujita, K. (1997) A Newly Developed Collagen/Silicone Bilayer Membrane as a Mucosal Substitute: a Preliminary Report. British Journal of Oral and Maxillofacial Surgery, 35, 85-91. https://doi.org/10.1016/S0266-4356(97)90681-1

[9] Ogasawara, T., Saito, I., Yamaguchi, M, Ioroi, K. and Shirakawa, M. (2003) Clinical Evaluation of Applying Atelocollagen Sponge to Protect Tooth Extraction Wound in Compromised Patients. Japanese Society of Oral Implantology, 16, 493-506. 
[10] Hirota, M., Mizuki, N., Aoki, S., Omura, S., Watanuki, K., Ozawa, T., Iwai, T., Matsui, Y. and Tohnai, I. (2009) Efficacy of Tooth Extraction Wound Protection Made of Atelocollagen Sponge (TRE-641): A Pilot Study in Dogs. Journal of Hard Tissue Biology, 18, 89-94. https://doi.org/10.2485/jhtb.18.89

[11] Kondo, S. and Kuroyanagi, Y. (2012) Development of Wound Dressing Composed of Hyaluronic Acid and Collagen Sponge with Epidermal Growth Factor. Journal of Biomaterials Science, 23, 629-643. https://doi.org/10.1163/092050611X555687

[12] Kondo, S., Niiyama, H., Yu, A. and Kuroyanagi, Y. (2012) Evaluation of a Wound Dressing Composed of Hyaluronic Acid and Collagen Sponge Containing Epidermal Growth Factor in Diabetic Mice. Journal of Biomaterials Science, 23, 1729-1740. https://doi.org/10.1163/092050611X597799

[13] Yu, A., Takeda, A., Kumazawa, K., Miyoshi, H., Kuroyanagi, M., Yoshitake, T., Uchinuma, E., Suzuki, R. and Kuroyanagi, Y. (2015) Preliminary Clinical Study Using a Novel Wound Dressing Composed of Hyaluronic Acid and Collagen Containing EGF. Open Journal of Regenerative Medicine, 4, 6-13. https://doi.org/10.4236/ojrm.2015.41002

[14] Kuroyanagi, Y., Ohno, H., Suzuki, R., Kuroyanagi, M. and Takeuchi, I. (2020) Design of Medical Devices Using Spongy Sheet Composed of Hyaluronic Acid and Collagen. Open Journal of Regenerative Medicine, 9, 71-84. https://doi.org/10.4236/ojrm.2020.92008

[15] Chen, W.Y.J. and Abatangelo, G. (1999) Functions of Hyaluronan in Wound Repair. Wound Repair and Regeneration, 7, 79-89. https://doi.org/10.1046/j.1524-475X.1999.00079.x

[16] Pardue, E.L., Ibrahim, S. and Ramamurthi, A. (2008) Role of Hyaluronan in Angiogenesis and Its Utility to Angiogenic Tissue Engineering. Organogenesis, 4, 203-214. https://doi.org/10.4161/org.4.4.6926

[17] Postlethwaite, A.E., Seyer, J.M. and Kang, A.H. (1978) Chemotactic Attraction of Human Fibroblasts to Type I, II, and III Collagens and Collagen-Derived Peptides. Proceedings of the National Academy of the Sciences of the United States of America, 78, 871-875. https://doi.org/10.1073/pnas.75.2.871

[18] Coln, D., Horton, J., Ogden, M.E. and Buji, L.M. (1983) Evaluation of Hemostatic Agents in Experimental Splenic Lacerations. American Journal of Surgery, 145, 256-259. https://doi.org/10.1016/0002-9610(83)90074-0

[19] Yoshizato, K., Taira, T. and Yamamoto, N. (1985) Growth Inhibition of Human Fibroblasts by Reconstituted Collagen Fibrils. Biomedical Research, 6, 61-71. https://doi.org/10.2220/biomedres.6.61

[20] Suzuki, R. and Kuroyanagi, Y. (2013) Safety and Utility of a PMMA-based Tissue Adhesive for Closure of Surgical Incision Wounds, Journal of Biomaterials Science, 24, 287-300. https://doi.org/10.1080/09205063.2012.690276

[21] Prato, G.P.P., Rotundo, R., Magnani, C. and Soranzo, C. (2000) Tissue-Engineering Technology for Gingival Augmentation Procedures: A Case Report. International Journal of Periodontics Restrative Dentistry, 20, 552-559.

[22] Prato, G.P.P., Rotundo, R. and Magnani, C., Soranzo, C., Muzzi, L. and Cairo, F. (2003) An Autologous Cell Hyaluronic Acid Graft Technique for Gingival Augmentation: A Case Series. Journal of Periodontology, 74, 262-267.

https://doi.org/10.1902/jop.2003.74.2.262

[23] Murata, M., Okuda, K., Momose, M., Kubo, K., Kuroyanagi, Y., Wolff, L.F. and Yoshie, H. (2008) Root Coverage with Cultured Gingival Dermal Substitute Composed of Gingival Fibroblasts and Matrix: A Case Series. International Journal of Periodontics Restorative Dentistry, 28, 461-467. 